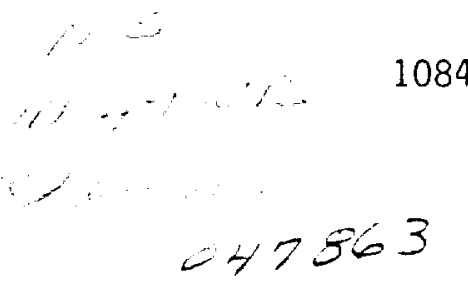

\title{
A Composite Diagnosis of Synoptic-Scale Extratropical Cyclone Development over the United States
}

\author{
Donald M. Rolfson* and Phillip J. Smith \\ Department of Earth and Atmospheric Sciences, Purdue University, West Lafayetre. Indiana
}

(Manuseript received 26 June 1995, in final form 15 November 1995)

\begin{abstract}
This paper presents a composite diagnosis of synoptic-scale forcing mechanisms associated with extratropical cyclone evolution. Drawn from 12 cyclone cases that occurred over the continental United States during the cool syclone evolution. Drawn from 12 cyclone cases that occurred over the continenthe the diagnosis provides a "climatology" of development mechanisms for difference categories of cyclone evolution ranging from cyclone weakening through three stages of cyclone intensification. Computational results were obrained using an "extended" form of the Zwack-Okossi equation applied to routine upper-air and surface data analyzed on a $230 \mathrm{~km} \times 230 \mathrm{~km}$ grid. Results show that cyclonic vorticity advection, which maximizes in the upper troposphere, was the primary contributor to cyclone development regardless of the stage of development. A second consistent contributor to development was latent heat release. Horizontal temperature advection, often acknowledged as a development mechanism, was found to contribute to develop. ment only during more intense stages. During weakening and weaker development stages, temperature advection menosed development, as the warm-air advection invariably found at upper levels was dominated by cold air advection in the lower half of the troposphere. In the more intense stages, development was moderated by dryadiabatic cooling associated with the ascending vertical motions.
\end{abstract}

\section{Introduction}

Studies of extratropical cyclone evolution have been for much of this century a major interest of the meteorological community, largely because they are the source of much of the inclement weather found in the midlatitudes and because, with their anticyclone partners, they are responsible for much of the potential to kinetic energy transformation found in the general circulation (Saltzman 1970). Dating from the work of the "Bergen school" in the 1910 s and 1920 s (e.g., Bjerknes 1919; Bjerknes and Solberg 1922), through the pioneering work of Sutcliffe (e.g., Sutcliffe 1939, 1947), and finally into contemporary interest in "explosive" cyclogenesis (e.g., Sanders and Gyakum 1980; Roebber 1984; Sanders 1986), a great deal has been learned about extratropical cyclone evolution. In some cases confirming previous understanding and in other cases providing new insight, recent papers demonstrate that cyclone development is often associated with

\footnotetext{
* Current affiliation; National Weather Scrvice Office. Rochester. MN.
}

Corresponding author address: Dr. Phillip J. Smith, Department of Earth and Atmospheric Sciences, Purdue University, 1397 Civil Engineering Building. West Lafayette, IN 47907-1397. cyclonic vorticity advection aloft (Sanders 1986; MacDonald and Reiter 1988); upper-tropospheric/ lower-stratospheric warm-air advection (Hirschberg and Fritsch 1991a,b; Lupo et al. 1992); latent heat release (Gyakum 1983a,b; Kuo and Reed 1988); surface energy fluxes (Nuss and Anthes 1987; Kuo et al. 1991); lowered static stability (Nuss and Anthes 1987; Smith and Tsou 1988); stratospheric extrusion and subsequent advection of high-potential-vorticity air (Uccellini et al. 1985; Elsberry and Kirchoffer 1988); and favorable jet streak positioning (Uccellini et al. 1984; Uccellini and Kocin 1987). Comprehensive reviews of cyclone studies can be found in Hoskins (1990), Uccellini (1990), and Bosart (1994).

This large array of antecedent studies has provided remarkable insight into cyclone development processes. However, while the basic cyclogenetic mechanisms may be well understood, the "mix" of these mechanisms during different stages of cyclone development is not always so clear. With this in mind, this paper describes a composite study of a set of forcing mechanisms that is sufficiently complete to account for the bulk of the synoptic-scale cyclone development process. The composites are drawn from 12 extratropical cyclones that occurred from late fall to early spring over the continental United States and extreme southern Canada. Using 
pressure tendencies diagnosed at the cyclone center to represent cyclone development, 54 individual map times represented by these cyclones were stratified (sample size in parenthesis) into weakening (8), weak development (9), moderate development (26), and strong development (11) categories. Diagnostic fields were then averaged for each category to provide composite values of forcing processes representative of different stages of cool-season cyclone evolution.

\section{The Zwack-Okossi equation}

The primary diagnostic tool used in this study is the Zwack-Okossi ( $Z-O$ ) equation, originally derived in quasigeostrophic form by Zwack and Okossi (1986) and recently redeveloped in a generalized form by Lupo et al. (1992). Combining the vorticity equation and a form of the first law of thermodynamics through the hydrostatic equation, the generalized $\mathrm{Z}-\mathrm{O}$ equation, given as Eq. (9) in Lupo et al. (1992), is

$$
\begin{aligned}
& \frac{\partial \zeta_{g l}}{\partial t}=\mathrm{PD} \int_{p_{t}}^{\rho_{t}} \cdot-\mathrm{V} \cdot \nabla \zeta_{p} d p-\mathrm{PD} \int_{p_{t}}^{p_{t}}\left[\frac{R}{f} \int_{p}^{\rho_{t}} \nabla^{2}\left(-\mathrm{V} \cdot \nabla T+\frac{\dot{Q}}{c_{p}}+S \omega\right) \frac{d p}{p}\right] d p+\mathrm{PD} \int_{p_{t}}^{p_{t}} \mathrm{k} \cdot \nabla \times \mathrm{F} d p \\
& \text { (A) } \\
& \text { (B) (C) (D) } \\
& +\mathrm{PD} \int_{p_{1}}^{p_{1}}-\frac{\partial \zeta_{28}}{\partial t} d p+\mathrm{PD} \int_{p_{1}}^{p_{t}}-\omega \frac{\partial \zeta_{a}}{\partial p} d p+\mathrm{PD} \int_{p_{1}}^{p_{1}}\left(-\frac{\partial \omega}{\partial x} \frac{\partial v}{\partial p}++\frac{\partial \omega}{\partial y} \frac{\partial u}{\partial p}\right) d p+\mathrm{PD} \int_{p_{t}}^{p_{1}} \zeta_{a} \frac{\partial \omega}{\partial p} d p
\end{aligned}
$$

(F)

(G)

$(\mathrm{H})$ where $p_{l}$ is a near-surface pressure level, $p_{t}$ is an upper pressure level sufficiently high to encompass most of the atmosphere, $\zeta_{g l}$ is the near-surface geostrophic vorticity, $\zeta_{a}$ is the absolute vorticity $(\zeta+f), f$ is the Coriolis parameter, $R$ is the dry-air gas constant, $V$ is the horizontal wind vector with components $u$ and $u, Q$ is the diabatic heating/cooling rate per unit mass, $c_{p}$ is the specific heat at constant pressure, $S$ is the static stability parameter [ $(T / \theta)(\partial \theta / \partial p)$, where $\theta$ is potential temperature $], \omega$ is the vertical motion in isobaric coordinates $(\omega=d p / d t), \mathrm{F}$ is the frictional force, and $\mathrm{PD}$ $=\left(p_{t}-p_{t}\right)^{-1}$.

Lupo et al. (1992) suggest that when synoptic-scale or larger phenomena are the object of the diagnosis it is appropriate to use the following simplified form of (1)

$$
\begin{array}{r}
\frac{\partial \zeta_{g l}}{\partial t}=\mathrm{PD} \int_{p_{t}}^{p_{t}}-\mathrm{V} \cdot \nabla \zeta_{c} d p-\mathrm{PD} \int_{p_{t}}^{p_{t}}\left[\frac{R}{f} \int_{p}^{p_{t}} \nabla^{2}\right. \\
(\mathrm{A}) \\
\left.\left(-\mathrm{V} \cdot \nabla T+\frac{\dot{Q}}{c_{p}}+S \omega\right) \frac{d p}{p}\right] d p,
\end{array}
$$

(C)

the so-called "extended" form of the Z-O equation. This simplified form is obtained from (1) by eliminating terms $F, G$, and $H$ based on scaling arguments similar to those applied to the height tendency equation of Tsou et al. (1987) and term I because it integrates to near zero as a result of compensating convergence/divergence. The frictional term $(E)$ was eliminated because it is normally a small contributor and because its computation requires making assumptions about surface conditions that are not known to any degree of certainty. Note that nonquasigeostrophic forcing is included in the right-hand-side terms. Also note that (2) is in a sufficiently simple form that the calculations for a particular map time can be accomplished quickly enough for near real-time diagnosis of cyclone events. Although not important for this paper, this feature has proven useful in the utilization of the $\mathrm{Z}-\mathrm{O}$ methodology in the classroom.

The reader may wonder why the $\mathrm{Z}-\mathrm{O}$ equation is used rather than other alternatives. Equation (2) is a generalization of the Petterssen-Sutcliffe equation (Petterssen 1956, 320-325) that permits explicit consideration of both dynamic and thermal forcing mechanisms at all atmospheric levels and includes nonquasigeostrophic forcing. Altemative equations that might be used for the diagnosis presented here either do not explicitly include forcing at all levels (e.g., PetterssenSutcliffe equation), do not explicitly isolate dynamic forcing mechanisms (e.g., Hirschberg-Fritsch equation; Hirschberg and Fritsch 1991 a,b), or do not lend themselves to easy isolation of the forcing contributions from each level (e.g., height tendency equation; Tsou et al. 1987). The integrands of the $p_{l}$ to $p_{t}$ integration are proportional to the horizontal divergence forced at each pressure level $p$ by horizontal vorticity advection ( $v a d v$, term $A$ ), horizontal temperature advection (tadv, term B), diabatic heating (only latent heat release in this study, lath, term C), and adiabatic cooling/warming (adia, term D).

Term A represents the change in near-surface geostrophic vorticity forced by divergence/convergence induced by ageostrophic motions that occur as the atmosphere attempts to reestablish geostrophic balance in response to local horizontal vorticity advection. Thus, for example, cyclonic vorticity advection at any 


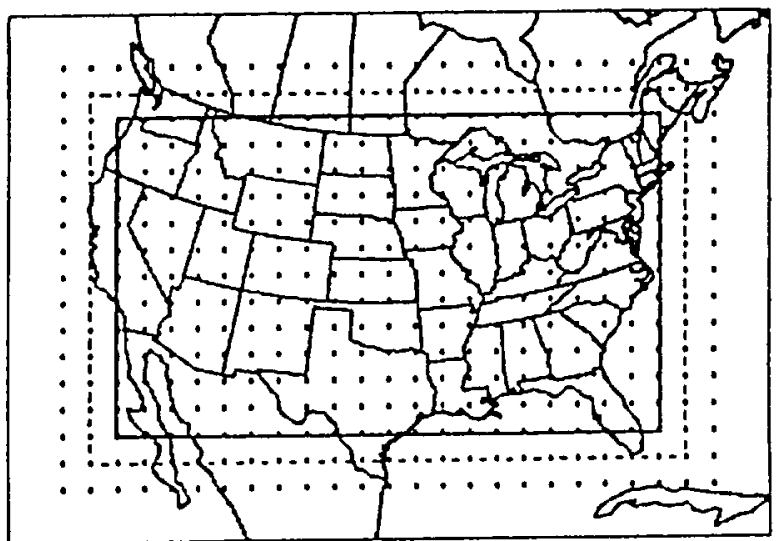

FiG. 1. Total analysis domain represented by all grid points. Computational (display) domain enclosed by dashed (solid) boundaries.

level above the surface will induce horizontal divergence at that level, which in tum will contribute to geostrophic vorticity (pressure) increases (decreases) at the surface. Terms $B$ and $C$ represent the horizontal divergence/convergence and change in near-surface geostrophic vorticity forced by integrated horizontal temperature advection and diabatic heating/cooling, respectively, from the surface to level $p$ (interior integral of these terms). For example, horizontally differential (given by the Laplacian operator), integrated warming from $p$, to $p$ will elevate the pressure levels at level $p$ and above. This in turn will force ageostrophic outflow (divergence) and surface vorticity (pressure) increases (decreases). The examples given for terms A-C also typically yield upward vertical motions. Such motions force dry adiabatic cooling, which in turn for a stable atmosphere $(S>0)$ yields a value for term $\mathrm{D}$ that is opposite to that of terms A-C. Thus, term D is often found to oppose the development characteristics of terms $A-C$.

\section{Computational features}

\section{a. Data, analyses, and finite differencing}

Routinely observed upper-air and surface data over the continental United States and surrounding regions were used in this study. The upper-air data were obtained from standard rawinsonde soundings taken twice daily at 0000 and 1200 UTC. From the sounding data, the surface pressure $(\mathrm{mb})$ and the geopotential heights $(\mathrm{m})$, temperatures $(\mathrm{C})$, wind speeds $(\mathrm{kt})$, and wind directions $\left({ }^{\circ}\right)$ at the mandatory reporting levels up to $50 \mathrm{mb}$ were retained for computations. From the surface data, sea level pressures (mb) at 0000, 0600, 1200, and 1800 UTC and 6-h precipitation accumulations [in. $(6 \mathrm{~h})^{-1}$ ] recorded at 0000 and 1200 UTC were retained. All data were obtained using the Purdue University Weather Processor (WXP), which has a routine



FIG. 2. Response function for Bames objective analysis.

to sort and format the data arriving through the domestic data circuit.

All surface and mandatory level data were fit to a 25 $\times 17$ polar-stereographic grid (Fig. 1) using a two-pass Bames objective analysis scheme (Barnes 1964, 1973). The polar stereographic grid is true at $60^{\circ} \mathrm{N}$ with a grid spacing of $230 \mathrm{~km} \times 230 \mathrm{~km}$. Application of the Barnes objective analysis requires the specification of two filter parameters, $C$ and $G$, which were chosen as 60000 and 0.8 , respectively. This provides a response function (Fig. 2) in which essentially no information is retained at wavelengths less than $500 \mathrm{~km}$, approximately $23 \%$ is retained at $1000-\mathrm{km}$ wavelengths, and $83 \%$ is retained at $2000-\mathrm{km}$ wavelengths. All calculations involved with gridding the data were performed through the algorithms contained in WXP. After the grids at the mandatory levels were created, these data were then interpolated linear in $\ln (p)$ to standard isobaric surfaces to form a set of data vertically distributed in 50-mb increments from 1050 to $50 \mathrm{mb}$. In performing the interpolation, all winds below ground were set to zero, and geopotential heights were calculated with the hypsometric equation using the interpolated temperatures. All horizontal derivative calculations at grid points along and within the interior solid box in Fig. 1 were determined with fourth-order finite differencing,

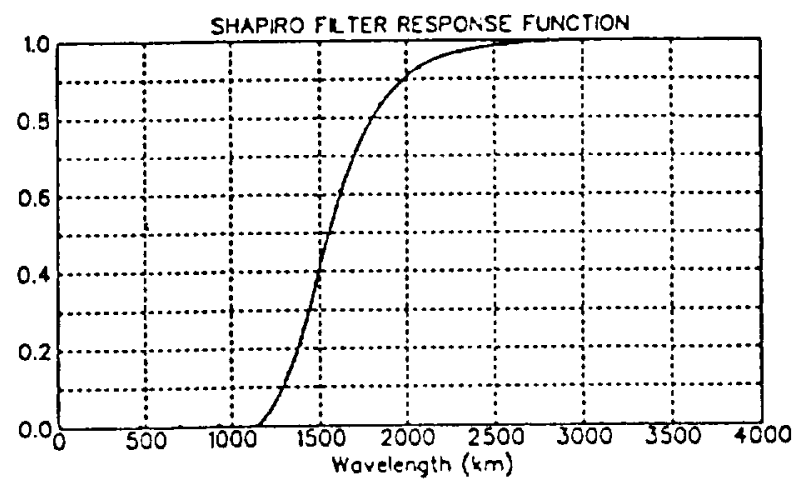

FIG. 3. Response function for Shapiro filtering procedure. 
while those along the dashed lines were determined with second-order finite differencing. Second-order finite differencing was also applied to all vertical derivatives. Vertical integrals were calculated using the trapezoidal rule.

\section{b. Vertical velocities}

Vertical velocities were calculated using the omega equation in the "extended" form as given by Tsou et al. (1987),

$$
\begin{array}{r}
{\left[\nabla^{2}+\frac{f^{2}}{\sigma}(\zeta+f) \frac{\partial^{2}}{\partial p^{2}}\right] \omega=\frac{f}{\sigma} \frac{\partial}{\partial p}\left(\mathrm{~V} \cdot \nabla \zeta_{a}\right)} \\
+\frac{R}{\sigma p} \nabla^{2}(\mathrm{~V} \cdot \nabla T)-\frac{R}{\sigma p} \nabla^{2}\left(\frac{\dot{Q}}{c_{p}}\right) .
\end{array}
$$

where $\sigma$ is the static stability parameter $[\sigma=-(R T)$ $P \theta)(\partial \theta / \partial p)]$. Sequential overrelaxation was used to solve ( 3 ) with the relaxation iteration being terminated when the absolute difference between two successive solutions of $\omega$ was less than $10^{-3} \mu \mathrm{b}^{-1}$ at all grid points. In the relaxation, the initial condition, the upper vertical boundary, and the lateral boundary conditions were set to zero. The lower vertical boundary value was specified as $-\mathrm{V} \cdot \nabla \rho_{s} g z$, where $z$ is the height of the terrain and $\rho_{s}$ is the surface air density calculated from the surface pressure and temperature. Vertical velocities for grid points below the earth's surface were assigned a value of zero.

\section{c. Diabatic heating}

In this study, the only diabatic heating contribution included was latent heat release (LHR). The LHR was calculated by taking the surface 6-h precipitation accumulation ending at each computational map time (0000 or 1200 UTC) and converting it to its equivalent integrated latent heating rate. The relationship relating precipitation rate to latent heating rate can be derived through the basic relationship

$$
\dot{Q}=-L \frac{d w_{s}}{d t},
$$

where $Q$ is the latent heating rate per unit mass, $L$ is the latent heat of vaporization, and $d w_{s} / d t$ is the rate of change of the saturation mixing ratio. From (4), the total column heating rate $(H)$ can be calculated by

$$
H=\frac{1}{\Delta p} \int \dot{Q} d p=-\frac{L}{\Delta p} \int \frac{d w_{s}}{d t} d p .
$$

where $\Delta p$ is the depth of the column in which the LHR is occurring. The precipitation rate $P$ can be expressed as

$$
P=-\int \frac{d w_{s}}{d t} \rho d z
$$

where $\rho$ is the air density. Applying the hydrostatic equation to (6) gives

$$
P_{g}=-\int \frac{d w_{s}}{d t} d p
$$

where $g$ is the acceleration due to gravity $\left(9.81 \mathrm{~m} \mathrm{~s}^{-1}\right)$. Finally, substitution of (7) into (5) yields

$$
H=\frac{g L P}{\Delta p} \text {. }
$$

The relationship given in ( 8 ) provides the means to convert a precipitation rate to an equivalent latent heating rate. This heating rate was then distributed in the vertical in the following manner:

(i) if the gridpoint surface pressure was $1000 \mathrm{mb}$ or greater, LHR was bounded (set equal to zero) at the lower and upper levels by 900 and $300 \mathrm{mb}$, respectively, with the maximum LHR at $700 \mathrm{mb}$;

(ii) if the gridpoint surface pressure was less than $1000 \mathrm{mb}$ but greater than or equal to $900 \mathrm{mb}$, LHR was bounded by 850 and $300 \mathrm{mb}$ with the maximum LHR at $700 \mathrm{mb}$;

(iii) if the gridpoint surface pressure was less than $900 \mathrm{mb}$ but greater than or equal to $850 \mathrm{mb}, \mathrm{LHR}$ was bounded by 800 and $300 \mathrm{mb}$ with the maximum LHR at $650 \mathrm{mb}$; and

(iv) if the gridpoint surface pressure was less than $850 \mathrm{mb}$, LHR was bounded by 750 and $300 \mathrm{mb}$ with the maximum LHR at $600 \mathrm{mb}$.

With this distribution method, no matter what the surface elevation, LHR began at approximately $50-$ $150 \mathrm{mb}$ above the surface with the maximum LHR placed approximately $200-300 \mathrm{mb}$ above the surface. The location of the LHR maximum is consistent with the mean latent heat release profiles reported for different cyclone intensities and development phases by MacDonald and Reiter (1988). Once the appropriate distribution method from above was chosen for a grid point, the LHR was then increased linearly in $\ln (p)$ from the lower bound to the level of maximum LHR and then decreased linearly in $\ln (p)$ from the level of maximum LHR to the upper bound.

To examine the sensitivity of the results to the vertical distribution of LHR, two alternative vertical distributions were tried for one map time, 1200 UTC 3 December 1990 , a time of significant LHR influence. One alternative was to place the maximum LHR $50 \mathrm{mb}$ above the lower bound (approximately 100-200 mb above the surface), while the other was to place the maximum $400 \mathrm{mb}$ above the lower bound (approximately 450 to $550 \mathrm{mb}$ above the surface). The upper and lower bounds were the same as determined in the original procedure. Considering the impact on both terms $C$ and $D$, the pressure tendency at the cyclone center was $8.5 \%$ larger for 



Fic. 4. (a) Central sea level pressures (mb) and (b) cyclone tracks for the 12 cyclone cases.

the lower maximum and $23 \%$ smaller for the higher maximum. Further, maps of pressure tendency (not shown) showed little change in the pattern and only small differences in magnitudes at most grid points. Thus, we conclude that the basic conclusions of this paper are not significantly influenced by the choice of LHR maximum level.

\section{d. Pressure tendency calculations}

The vertical computational domain extended from a near-surface level $\left(p_{l}\right)$, defined as the first pressure level above the surface identically divisible by 50 ( 950 or $900 \mathrm{mb}$ at most grid points) to $50 \mathrm{mb}\left(p_{t}\right)$. Forcing quantities in (2) were calculated at each level and then 



Fig. 4. (Continued)

integrated to yield geostrophic vorticity tendencies at level $p_{l}$. At this level, the tendencies are always above the surface but are considered close enough (from 0 to $49 \mathrm{mb}$ above the surface) to be representative of actual surface development. After the geostrophic vorticity tendency field was calculated for each term in (2), each term was filtered using a two-dimensional, fourth-order
Shapiro (1970) filtering scheme. Filtering was necessary to remove subsynoptic "noise" introduced through finite differencing in the basic calculations. The response function for this filter (Fig. 3) retains essentially zero information at wavelengths less than $1000 \mathrm{~km}$, approximately $40 \%$ at $1500 \mathrm{~km}$, and $90 \%$ at $2000 \mathrm{~km}$. Prior to filtering, the gridpoint values along 



FIG. 4. (Continued)

the dashed lines shown in Fig. 1 were used to expand the grid by two rows at the top and bottom and two columns on each side. For example, the values at the grid points along the top row dashed line were used as the values for the grid points in the new row above the dashed line. This procedure was performed on all sides of the grid using the gridpoint values along the other dashed lines. Each of the four comer points was set to the gridpoint value diagonal from it. After this first expansion, the procedure was performed once more using the values at the grid points determined from the first expansion. Expanding the grid was necessary in order 


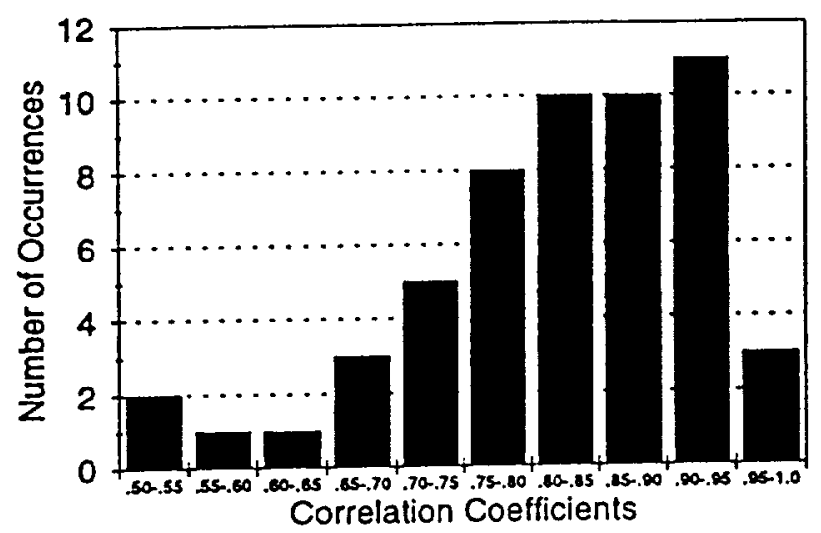

FIG. 5. Distribution of correlation coefficients between Z-O and observed pressure tendencies for all map times.

to reduce the boundary effects, which can pose problems with small grids like the one used in this study.

The filtered geostrophic vorticity tendencies were summed to provide the total $\mathrm{Z}-\mathrm{O}$ geostrophic vorticity tendency field at $p_{1}$. Then, sequential overrelaxation was used to obtain the total $\mathrm{Z}-\mathrm{O}$ near-surface height tendency field. In this relaxation, the initial condition was set to zero, while the lateral boundaries were set to the observed 6-h height tendencies, computed from the present minus previous 6 -h sea level pressure values using $\Delta z / \Delta t=\left(1 / \rho_{s} g\right)\left(\Delta p_{\mathrm{SL}} / \Delta t\right)$, where $p_{\mathrm{SL}}$ is the sea level pressure. Relaxation continued until the absolute difference between two successive solutions of height tendency was less than $10^{-5} \mathrm{~m} \mathrm{~s}^{-1}$ at all grid points. The final $\mathrm{Z}-\mathrm{O}$ height tendency field was then converted to a surface pressure $p_{\text {s }}$ tendency field using $\left(\partial p_{s} / \partial t\right)=\rho_{s} g\left(\partial z_{l} / \partial t\right)$.

\section{e. Compositing procedure}

Compositing was done by averaging $\mathrm{Z}-\mathrm{O}$ near-surface geostrophic vorticity and surface pressure tendencies and vertical profiles of relevant parameters at the cyclone center (the grid point with lowest sea level pressure) for each development category. The center point was used because it is an easily identified point common to all cyclones and because at this point the propagation component of the pressure tendency is equal to zero. Thus, the center point pressure tendency corresponds to the quasi-Lagrangian pressure change or to the development rate.

Individual map times of cyclone development were stratified into four development categories based on the center-point $\mathrm{Z}-\mathrm{O}$ surface pressure tendencies at each time. The four categories are defined as weakening (WK, $\partial p_{s} / \partial t>0$ ); weak development [WD, $0 \geqslant \partial p_{s} /$ $\partial t>-4 \mathrm{mb}(12 \mathrm{~h})^{-1}$ ]; moderate development [MD, $\left.-4 \mathrm{mb}(12 \mathrm{~h})^{-1} \geqslant \partial p_{s} / \partial t>-8 \mathrm{mb}(12 \mathrm{~h})^{-1}\right]$ (approximately $0.5-0.9$ bergerons); and strong develop-

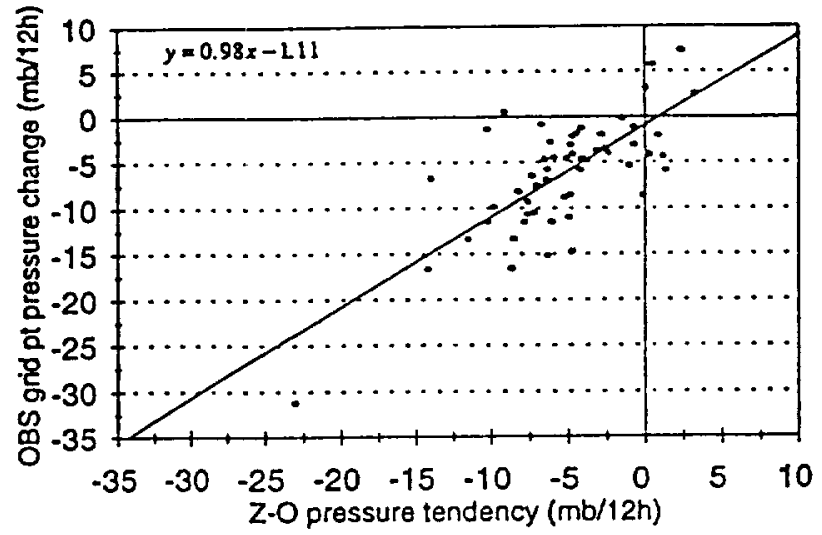

FiG. 6. The $\mathrm{Z}-\mathrm{O}$ versus observed pressure tendencies at the grid point of the cyclone center for all map times. The equation for simple linear regression is provided on the plot.

ment $\left[S D,-8 \mathrm{mb} \geqslant \partial p_{s} / \partial t(12 \mathrm{~h})^{-1}\right]$ (approximately 0.9 bergerons and greater). Using this classification method, 8, 9, 26, and 11 map times were included in the WK, WD, MD, and SD groups, respectively. Results are presented as center-point averages over each development category.

\section{Cyclone cases}

Examined in this study were the 12 cyclones identified by the cyclone tracks and central pressures shown in Fig. 4. These cases were chosen to provide a representative sample of typical cool season cyclones that included examples of all of the development categories and were located in the interior of the data network. Examination of the central sea level pressure of each cyclone indicates phases of development ranging from weakening to explosive intensification. Four cyclones-cases 03DEC90, 02NOV92,12NOV93, and 24MAR94 - clearly exhibited both a period of deepening and a period of weakening. Two cyclones cases 12 NOV 93 and 26JAN94 - experienced brief periods $(6 \mathrm{~h})$ of explosive development at rates of 1.3 and 1.1 bergerons, respectively, while case 24MAR94 developed explosively for $18 \mathrm{~h}$ at a rate of 1.1 berge-

TABLE 1. Mean absolute values [MAV, mb $(12 h)^{-1}$ ] and correlation coefficients (COR) for the four development classes. DIFF represents the percentage difference between OBS and $Z-O$ MAV values, that is, [(Z-O MAV) - (OBS MAV)]/(OBS MAV) $\times 100$.

\begin{tabular}{lcccc}
\hline & WK & WD & MD & SD \\
\hline OBS MAV & 4.20 & 5.24 & 4.51 & 6.02 \\
Z-O MAV & 3.55 & 3.84 & 3.99 & 6.05 \\
DIFF & -15 & -27 & -12 & 0.5 \\
COR & 0.77 & 0.81 & 0.82 & 0.83 \\
\hline
\end{tabular}



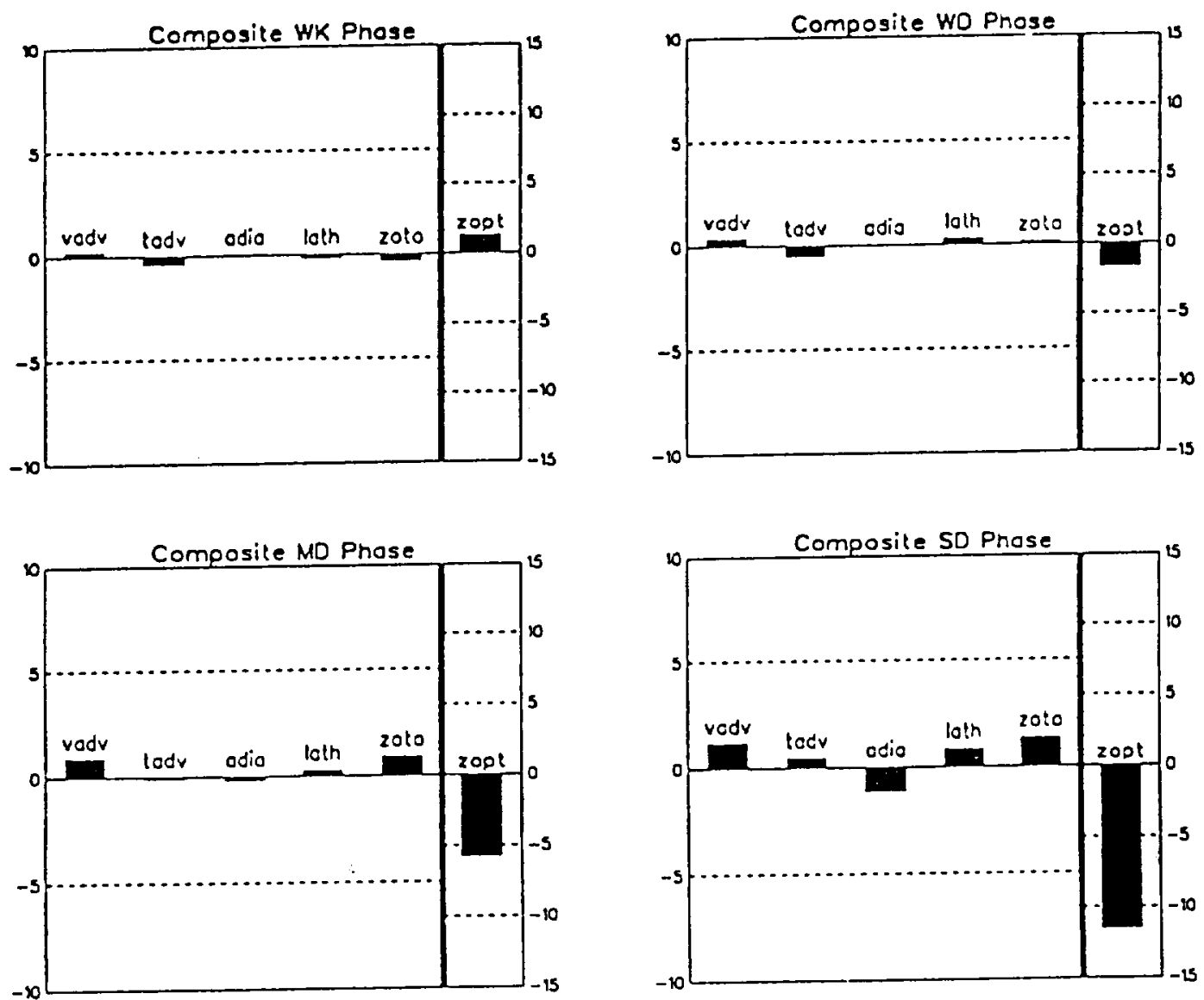

FIG. 7. Composite near-surface geostrophic vorticity tendencies $\left(10^{-9} \mathrm{~s}^{-2}\right)$ at the cyclone center forced by the vorticity advection term (vadv), temperature advection term (tadv), adiabatic term (adia), and latent heating term (lath); the total $\mathrm{Z}-\mathrm{O}$ near-surface geostrophic vorticity tendency (zoto); and the $\mathrm{Z}-\mathrm{O}$ surface pressure tendency [zopt, mb $(12 \mathrm{~h})^{-1}$ ] for each of the four development categories.

rons. Only one case, 12NOV92, experienced an extended explosive development period that lasted at least 24 h. During the period 0600 UTC 12 November -0600 UTC 13 November, the cyclone intensified at a rate of 1.9 bergerons. The other cyclone cases exhibited various degrees of nonexplosive deepening with some periods of weakening.

\section{Results}

\section{a. Comparisons of $Z-O$ and observed pressure tendencies}

The Z-O surface pressure tendencies were compared with observed (OBS) sea level pressure changes, computed from observed sea level pressure at the diagnosis time minus observed sea level pressure $6 \mathrm{~h}$ earlier. Adoption of the backward 6-h time difference was dictated by classroom use of this methodology for realtime cases. This provides us with an observed tendency field that is available for comparison with the $\mathrm{Z}-\mathrm{O}$ ten- dencies as soon as they are calculated. The correlation coefficients (COR) for all map times in this study (Fig. 5) suggest very favorable pattern comparability between the two fields. In fact, 34 of the 54 map times had COR values exceeding 0.8 , and 14 of those values were above 0.9 . The worst and best $C O R$ values were 0.50 and 0.96 , respectively, and the average was 0.81 . Furthermore, in comparisons of Z-O pressure tendencies to OBS pressure changes at the gridpoint locations of the cyclone center (Fig. 6), there were only five occurrences of $\mathrm{Z}-\mathrm{O}$ and $\mathrm{OBS}$ tendencies having opposite sign, and in each of those five, one of the tendencies was always near zero. This suggests that the $\mathrm{Z}-\mathrm{O}$ equation provided a good diagnosis of the forcing processes leading to surface pressure changes, particularly near the cyclone center. Closer examination of Fig. 6 reveals that the $\mathrm{Z}-\mathrm{O}$ tendencies were biased toward values smaller than observed. Finally, the mean absolute values (MAV), averaged over all grid points and map times, also suggest good comparability between the two fields. The MAV for the $\mathrm{Z}-\mathrm{O}$ pressure 

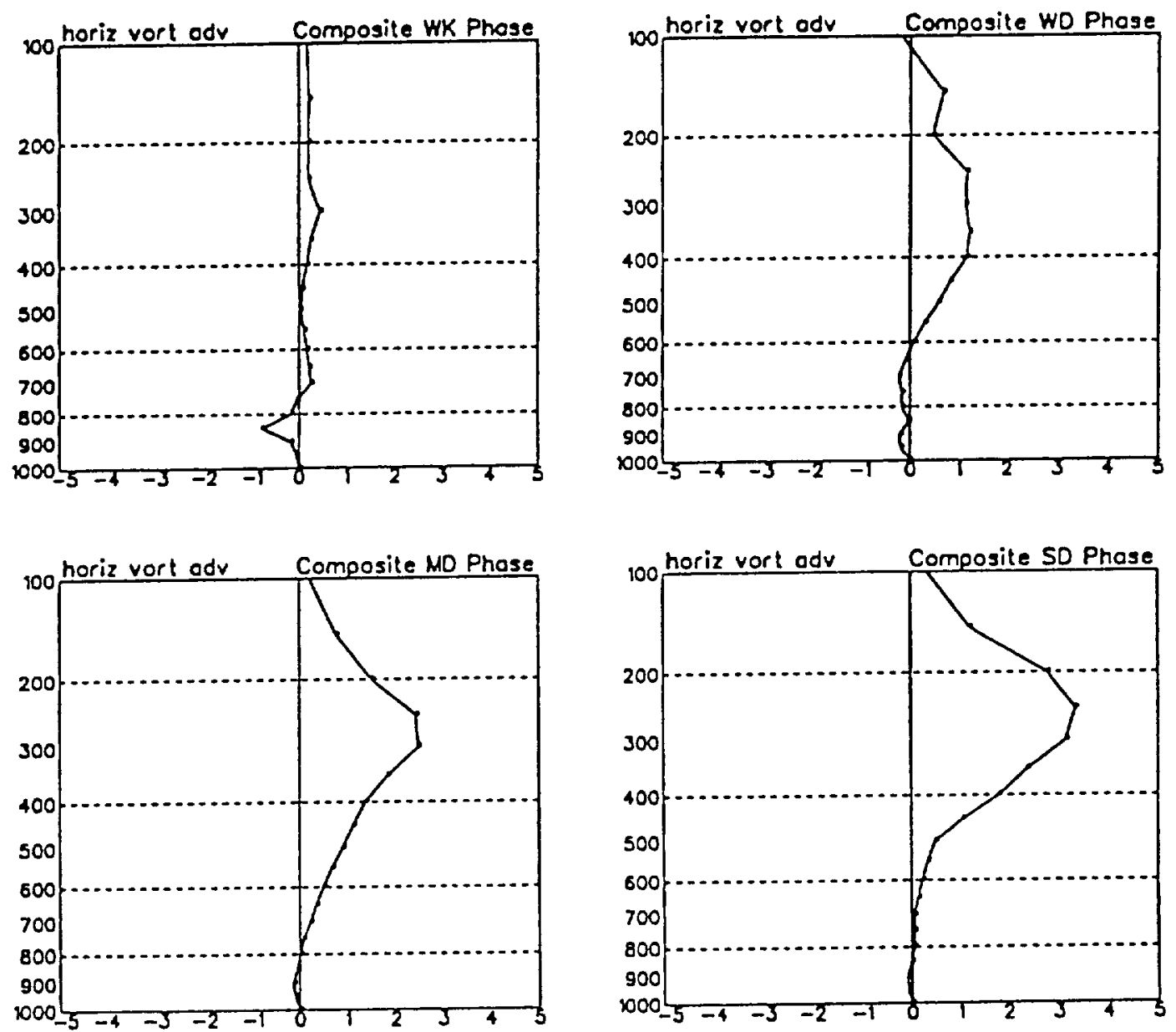

FIG. 8. Composite vertical profiles of horizontal absolute vorticity advection $\left(10^{-9} \mathrm{~s}^{-2}\right)$ at the cyclone center for each of the four development categories.

tendencies, at $4.31 \mathrm{mb}(12 \mathrm{~h})^{-1}$, was only $12 \%$ smaller than the OBS MAV, at $4.89 \mathrm{mb}(12 \mathrm{~h})^{-1}$. Thus, while the COR values suggest good comparability of pressure fall and pressure rise regions between the $\mathrm{Z}-\mathrm{O}$ and OBS fields, the similar MAV's suggest good comparability between the magnitudes in the pressure fall and pressure rise regions.

Table 1 shows the comparison statistics, computed as averages, between the $\mathrm{Z}-\mathrm{O}$ and $\mathrm{OBS}$ pressure tendencies in each of the four development categories. The WK phase had the lowest COR value, while the SD phase had the best COR value, as well as the smallest difference between OBS and Z-O MAV values at $0.5 \%$. The WD phase had the largest difference in MAV values, with the $Z-O$ being $27 \%$ smaller than the OBS. In general, the comparisons between the $\mathrm{Z}-$ $O$ and $O B S$ pressure tendency fields became more favorable with stronger development. This suggests that the $\mathrm{Z}-\mathrm{O}$ model works best when the synoptic-scale flow regime is active with prominent, intensifying cyclones.

\section{b. Composite $Z-O$ tendencies}

Figure 7 shows the composite near-surface geostrophic vorticity tendencies at the cyclone center point forced by each term in (2), as well as the total near-surface vorticity tendency (zoto) and the Z-O surface pressure tendency (zopt) for each development category. As expected, zoto (zopt) was negative (positive) for weakening cyclones and increas. ingly positive (negative) for increasingly stronger cyclone development.

The most consistent contributor to development was the vorticity advection, which monotonically increased in magnitude for stronger development categories. Failure of cyclones to intensify during the weakening periods could be largely attributed to the temperature advection influence, which made the largest negative contribution. This negative contribution also occurred during weak development periods and would have resulted in cyclone weakening except for the added positive influence of latent heat release. For both of these 



FiG. 9. As in Fig. 8 except for horizontal temperature advection $\left(10^{-4} \mathrm{~K} \mathrm{~s}^{-1}\right)$.

phases, dry-adiabatic processes had little impact on development.

During moderate development, the temperature advection influence was nearly zero, while the latent heat release influence remained nearly the same as during weak development. Adiabatic cooling assumed a stronger role, but its negative influence was more than compensated by the vorticity advection. Finally, strong development is marked by increased positive contributions by the dominant vorticity advection as well as temperature advection and latent heat release, which together were able to more than offset the increased adiabatic cooling influence. This latter result is qualitatively similar to the results of Lupo et al. (1992) for cases of marine and land explosive cyclogenesis.

\section{c. Vertical profiles}

Insight into the physical mechanisms responsible for the preceding results is best achieved by examining vertical profiles of the basic physical process repre- sented in each term, that is, horizontal vorticity advection, horizontal temperature advection, latent heat release, and adiabatic temperature change $(S \omega)$. Composite profiles for each development category are displayed in Figs. 8-13.

The composite profiles of horizontal absolute vorticity advection are shown in Fig. 8. The profiles for the WD, MD, and SD categories show a cyclonic vorticity advection (CVA) maximum in the upper troposphere, where the strongest winds are found, with the maximum becoming more prominent and well defined with increasing intensity of surface development. At low levels, weak anticyclonic vorticity advection (AVA) is seen for these three composites, with magnitude and vertical depth of influence that decreased as surface development increased. These profiles are similar to those reported in MacDonald and Reiter's (1988) composite study. Also, the SD profile is similar to the vorticity advection profiles in Lupo et al. (1992). The WK composite showed weak vorticity advections at all levels with no prominent CVA or AVA maximum. How- 

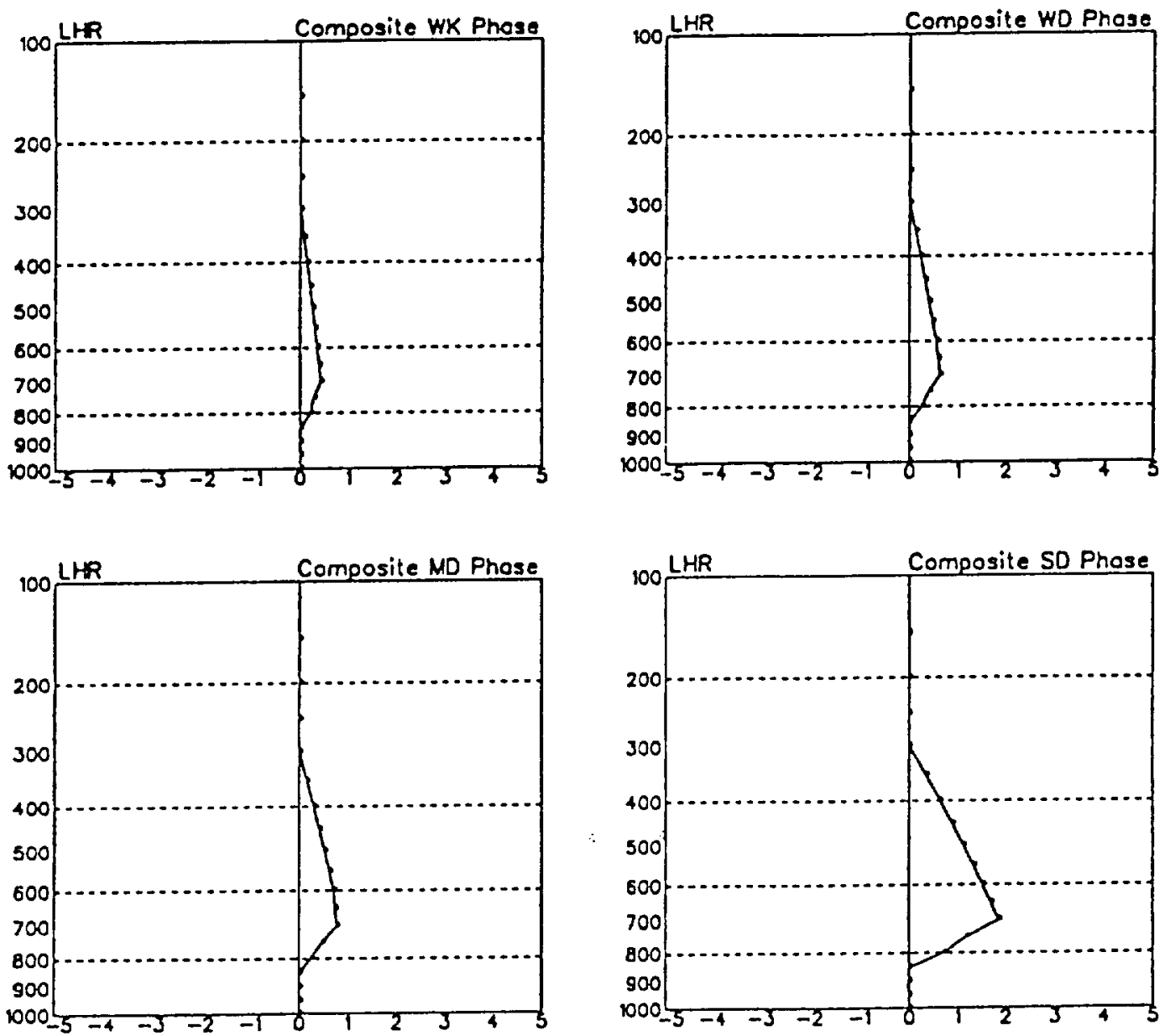

FiG. 10. As in Fig. 8 except for latent heat release $\left(10^{-4} \mathrm{~K} \mathrm{~s}^{-1}\right)$.

ever, similar to the other categories, CVA was found at all but the lower portion of the troposphere. Note the good correspondence between increasing CVA in the four categories and the increasing near-surface geostrophic vorticity tendency values contributed by vadv in Fig. 7.

Similar to vorticity advection, temperature advection profiles show a warm-air advection (WAA) maximum at upper atmospheric levels (Fig. 9), a common feature of cyclone development emphasized by Hirschberg and Fritsch (1991b) and confirmed by Lupo et al. (1992). Notice that as the degree of surface development increased in the four composites, the value of the upperlevel WAA maximum also increased. Also, in the MD and SD composites, a secondary WAA maximum was located in the lowest 150 to $200 \mathrm{mb}$, similar to the results of Lupo et al. (1992). Although WAA increased with increased surface development, it became dominant only in the SD composite, when as shown in Fig. 7 it forced near-surface geostrophic vorticity increases. In all four classes, cold-air advection (CAA) was found from 750 to $450 \mathrm{mb}$, its magnitude decreasing as surface development increased.

The composite profiles of LHR are shown in Fig. 10. The vertical distribution of LHR appears as specified in section $3 c$, with composite maxima at $700 \mathrm{mb}$ for all development classes. The profiles show that the LHR values increased steadily from the WK to the SD category. This corresponds to the increasing positive near-surface vorticity tendencies contributed by lath seen in Fig. 7. The small negative value contributed by lath seen in Fig. 7 for the WK category is a feature that often occurs in the finite-difference estimate of the laplacian when the cyclone center is located near the boundary of the latent heating region, particularly when the latent heating is small as is the case here. Although a consequence of the finite differencing, this result simulates the upper-air convergence (and hence surface geostrophic vorticity decrease) that would be expected in regions adjacent to the upper-air divergence area attributed to latent heat release. 

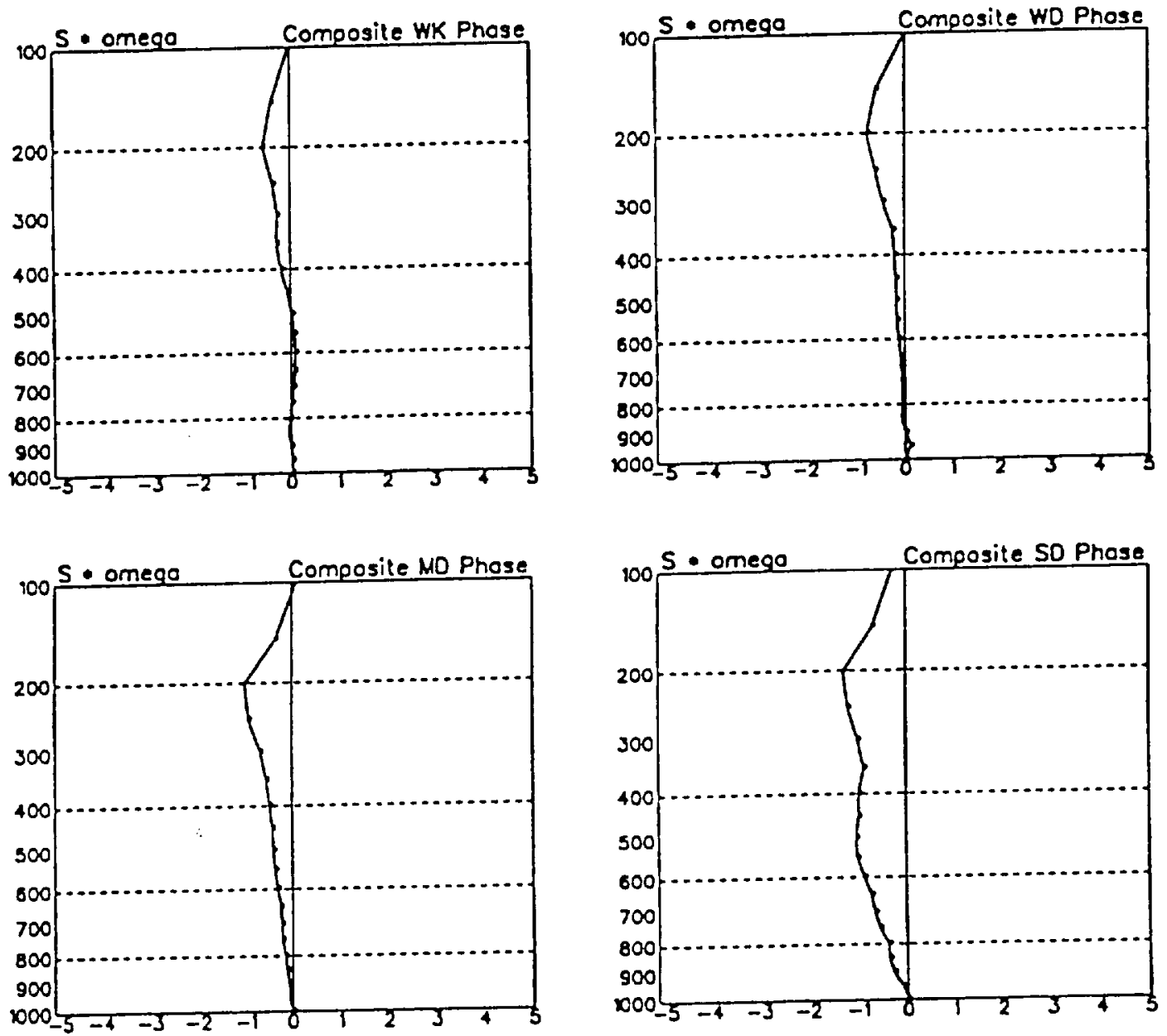

FIG. 11. As in Fig. 8 except for adiabatic heating-cooling $\left(10^{-1} \mathrm{~K} \mathrm{~s}^{-1}\right)$.

The composite profiles of $d r y$ adiabatic temperature change $(S \omega)$ are shown in Fig. 11. Because of the adiabatic cooling that they represent, these profiles are generally of opposite sign to the other thermodynamic profiles. In all categories, the magnitude of $S \omega$ maximizes in the upper troposphere at $200 \mathrm{mb}$. This is a result of sufficiently strong upward vertical motions (Fig. 12) occurring in the presence of increasing $S$ values (Fig. 13). The SD composite shows a secondary maximum at $500 \mathrm{mb}$, resulting from the upward vertical motion that maximizes at about this level. The only positive $S \omega$ values occurred in the WK composite from 700 to $500 \mathrm{mb}$, forced by downward vertical motions (Fig. 12). The increasing magnitude of the negative $S \omega$ cooling with increasing surface development corresponds to the increasing magnitude of the negative near-surface geostrophic vorticity tendencies contributed by adia seen in Fig. 7.

Composite vertical motion profiles (Fig. 12) indicate, as expected, stronger upward vertical motions for more intense development categories. The static sta- bility profiles (Fig. 13) show hydrostatically stable air at all levels with only minor changes with pressure until about $300 \mathrm{mb}$, above which $S$ increases rapidly. Minimum values are seen below $850 \mathrm{mb}$ during the WK. WD, and MD categories. However, a small, secondary maximum occurs in this layer during strong development. These differences in low'-level $S$ values suggest that if smaller low-level static stability is a favorable condition for cyclone development, then the smaller values must exert their influence prior to the onset of strong development. Stability values after strong development onset are actually relatively large. This result is similar to that reported by Smith and Tsou (1988) for a single cyclone case.

\section{Summary and conclusions}

A diagnosis of 12 cyclones that occurred over the continental United States and extreme southern Canada was performed using near-surface geostrophic vorticity tendencies and pressure tendencies derived from an 

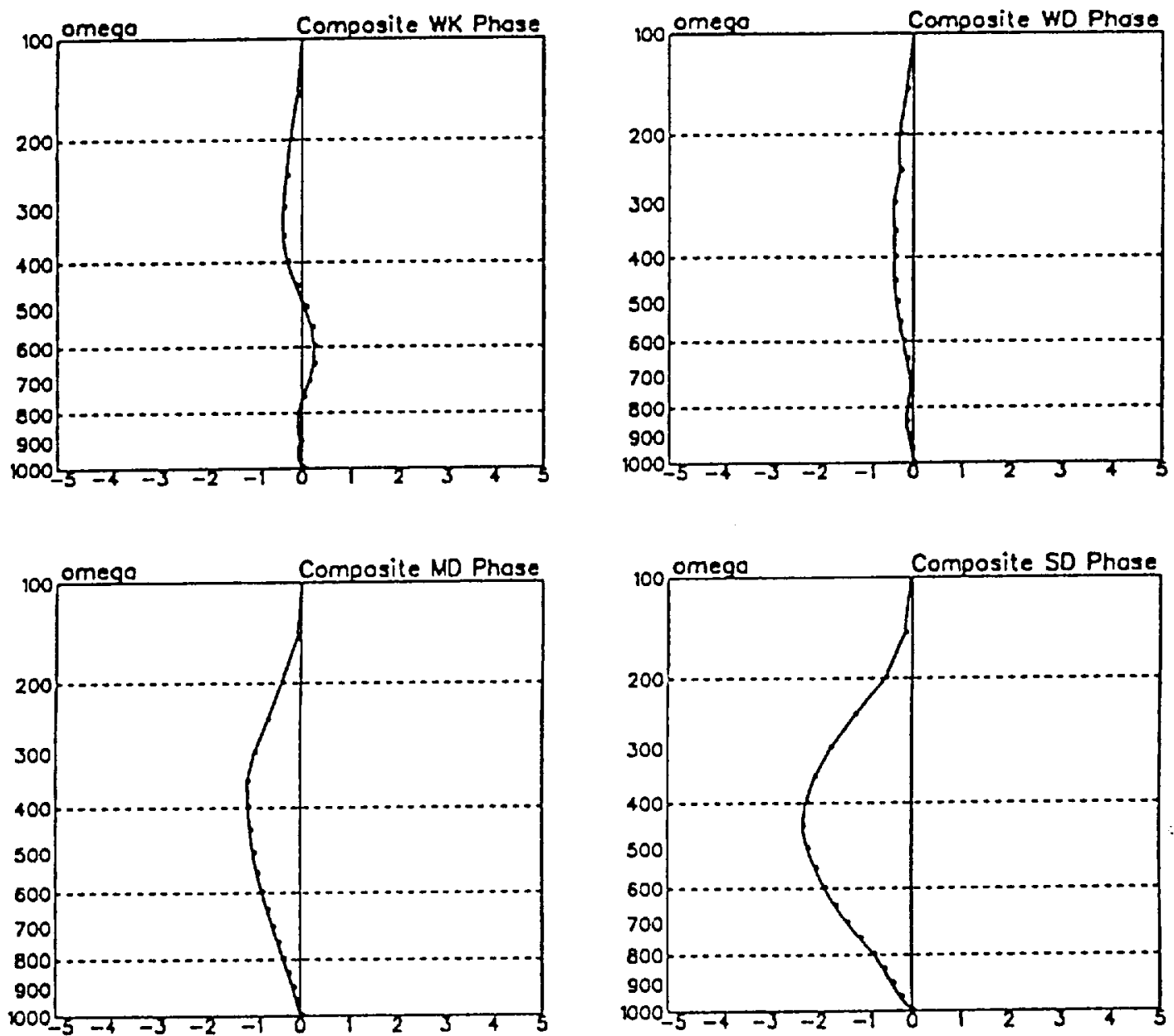

FiG. 12. As in Fig. 8 except for verical motion ( $\mu \mathrm{b} \mathrm{s}^{-1}$ ).

"extended" form of the Zwack-Okossi ( $Z-O)$ equation. Pressure tendencies obtained by relaxation of the total vorticity tendencies were compared to observed sea level pressure changes over the 6 -h periods ending at each diagnosis time. The comparisons between the two fields were very good, as suggested by the average correlation coefficient of 0.81 and their comparable magnitudes.

To examine the forcing processes influencing development in the 12 cyclones, composites were generated by placing the diagnostic results for each map time into one of four categories of development based on the Z$O$ surface pressure tendencies at the grid point of the cyclone center. The results show that cyclonic vorticity advection was the main contributor to surface development regardless of the cyclone development rate. This cyclonic vorticity advection was dominant in the upper troposphere and became more prominent as the strength of surface development increased. The main opposition to development in the weakening and weakly developing categories was provided by tem- perature advection, as cold-air advection in the lower half of the troposphere dominated upper-level warmair advection. Temperature advection contributed to development only during strong development, when warm-air advection that maximized in the upper troposphere was accompanied by a secondary maximum in the lower troposphere and the midtropospheric coldair advection decreased in magnitude. Adiabatic processes provided the main opposition to development in the periods of moderate and strong cyclone deepening. while latent heat release provided increasingly positive contributions as the degree of surface development increased. Only during strong development did vorticity advection, temperature advection, and latent heating all contribute to development.

Based on these results we are led to the expected conclusion that cyclonic vorticity advection and warmair advection provide upper-level support for cyclogenesis during all phases of cyclone evolution. However, somewhat surprisingly, despite the persistent upperlevel warm-air advection, vertically integrated temper- 

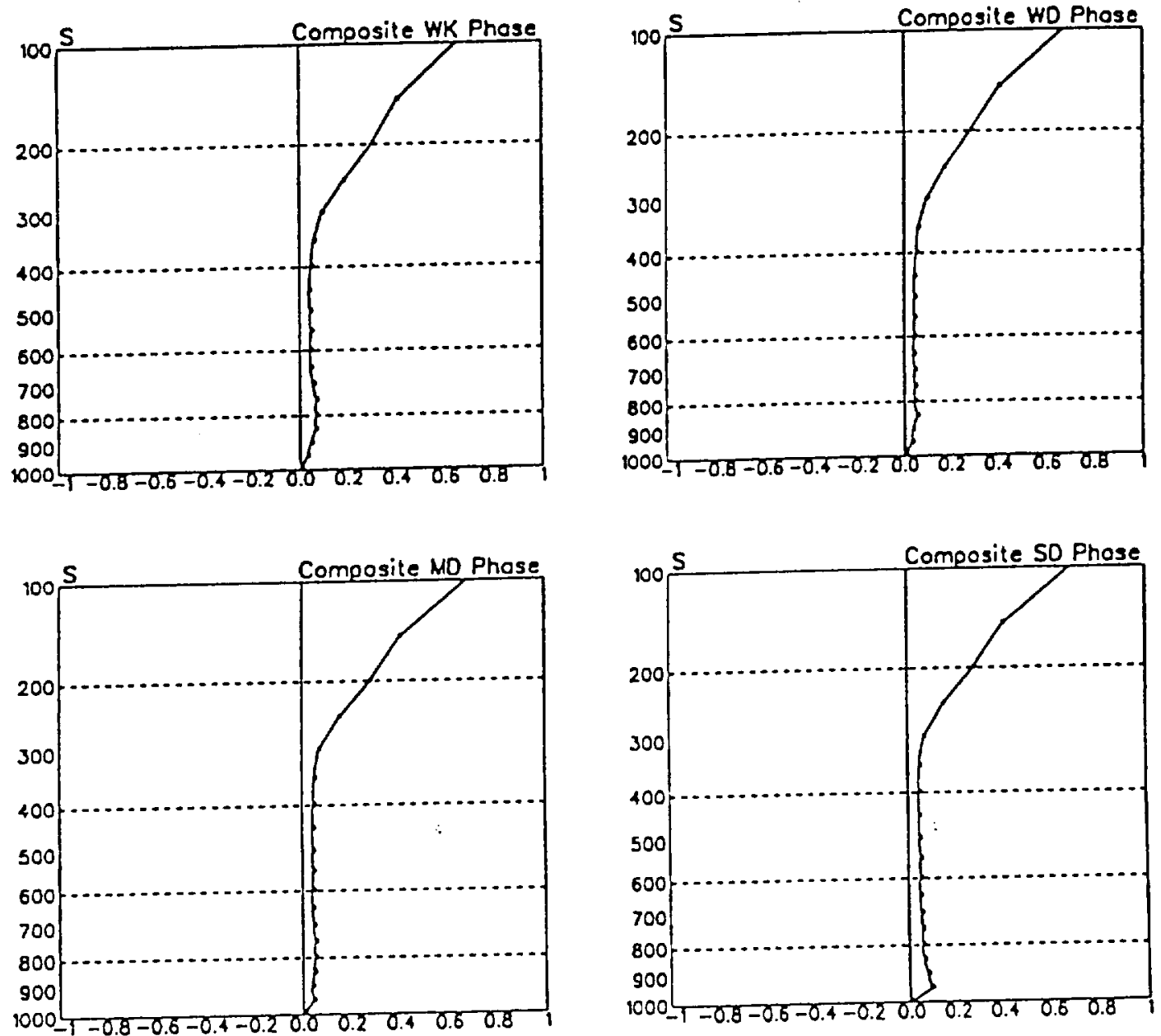

FIG. 13. As in Fig. 8 except for the static stability parameter $\left(K \mathrm{mb}^{-1}\right)$.

ature advection supports development only during strong development periods. This stands in contrast to vertically integrated vorticity advection and latent heat release, which are persistent cyclogenetic mechanisms. Finally, transitions from weaker to stronger development categories require that the total cyclogenetic mechanisms overcome the cyclolytic influence of dryadiabatic cooling. This cooling increases as the cyclone intensifies in association with increased upward vertical motions and, in later stages, increased static stability. The ability to overcome this increased cooling is realized as 1) integrated cyclonic vorticity advection and latent heat release increase and 2) upper-level warmair advection is joined by a secondary warm-air advection maximum in the lower troposphere, thus negating the inhibiting influence of the low to midlevel cold-air advection that dominates this term during weaker development stages.

Acknowledgments. We are grateful to Dayton Vincent, Robert Oglesby, Daniel Vietor, Anthony Lupo, and Robert (Marty) Rausch for their suggestions and assistance during the course of this work and to Helen Henry for typing the manuscript. This research was partially supported by the National Aeronautics and Space Administration under Grant NAG8-915.

\section{REFERENCES}

Barnes, S. L., 1964: A technique for maximizing details in numerical weather map analysis. J. Appl. Meteor., 3, 396-409.

1973: Mesoscale objective map analysis using weighted time series observations. NOAA Tech. Memo. ERL NSSL-62, 60 pp. [NTIS COM 73-10781.]

Bjerknes, J., 1919: On the structure of moving cyclones. Geofys. Publ., 1, 1-8.

- and $\mathrm{H}$. Solberg. 1922: Life cycle of cyclones and the polar front theory of atmospheric circulation. Geofys. Publ., 3, 1-18.

Bosart, L. F., 1994: Observed cyclone life cycles. Proc. of the Int. Symp. on the Life Cycles of Extratropical Cyclones, Bergen, Nonway, Geophysical Institute. University of Bergen. $111-148$.

Elsberty, R. L., and P. J. Kirchoffer, 1988: Upper-level forcing of explosive cyclogenesis over the ocean based on operationally analyzed fields. Wea. Forecasting. 3, 205-216. 
Gyakum, J. R., 1983a: On the evolution of the QE II Storm. Part I: Synoptic aspects. Mon. Wea. Rev., 111, 1137-1155.

_. 1983b: On the evolution of the QE II Storm. Part II: Dynamic and thermodynamic structure. Mon. Wea. Rev., 111, 11561173.

Hirschberg, P. A., and J. M. Fritsch. 1991 a: Tropopause undulations and the development of extratropical cyclones. Part I: Overview and observations from a cyclone event. Mon. Wea. Rev., 119, $496-517$.

_ and 1991b: Tropopause undulations and the development of extratropical cyclones. Part II: Diagnostic analysis and conceprual model. Mon. Wea. Rev., 119, 518-550.

Hoskins, B. J., 1990: Theory of extratropical ryclones. Extratropical Cyclones. The Erik Palmén Memorial Volume, C. W. Newton and E. O. Holopainen, Eds., American Meteorological Society, 64-80.

Kuo, Y. H., and R. J. Reed, 1988: Numerical simulation of an explosively deepening cyclone in the eastem Pacific. Mon. Wea. Rev., 116, 2081-2105.

_ and S. Low-Nam, 1991: Effects of surface energy fluxes during the early development and rapid intensification stages of seven explosive cyclones in the western Atlantic. Mon. Wea. Rev., 119, 457-476.

Lupo, A. R., P. J. Smith, and P. Zwack, 1992: A diagnosis of the explosive development of two extratropical cyclones. Mon. Wea. Rev., 120, 1490-1523.

MacDonald, B. C., and E. R. Reiter, 1988: Explosive cyclogenesis over the eastern United States. Mon. Wea. Rev.. 116, 15681586.

Nuss, W. A., and R. A. Anthes, 1987: A numerical investigation of low-level processes in rapid cyclogenesis. Mort. Wea. Rev., 115, :2728-2743.

Petterssen, S., 1956: Weather Analysis and Forecasting. Vol. I. 2d ed. McGraw-Hill, 428 pp.

Roebber, P. J., 1984: Statistical analysis and updated climatology of explosive cyclones. Mon. Wea. Rev., 112, 1577-1589.
Saluman, B. 1970: Large-scale atmospheric energetics in the wavenumber domain. Rev. Geophys. Space Phys., 8, 289-302.

Sanders, F. 1986: Explosive cyclogenesis over the west central North Atlantic Ocean 1981-1984. Par I. Composite structure and mean behavior. Mon. Wea. Rev., 114, 1781-1794.

—, and J. R. Gyakum, 1980: Synoptic-dynamic climatology of the "bomb." Mon. Wea. Rev., 108, 1589-1606.

Shapiro, R., 1970: Smoothing, filtering, and boundary effects. Rev. Geophys., 8, 359-387.

Smith. P. J., and C.-H. Tsou, 1988: Static stability variations during the development of an intense extratropical cyclone. Mon. Wea. Rev. 116, 1245-1250.

Sutcliffe, R. C. 1939: Cyclonic and anticyclonic development. Quart. J. Roy. Meteor. Soc. 65, 518-524.

1947: A contribution to the problem of development. Quart. J. Roy. Meteor. Soc., 73, 370-383.

Tsou, C.-H., P. J. Smith, and P. M. Pauley, 1987: A comparison of adiabatic and diabatic forcing in an intense extratropical cyclone system. Mon. Wea. Rev. 115, 763-786.

Uccellini, L. W. 1990: Processes contributing to the rapid development of extratropical cyclones. Extratropical Cyclones. The Erik Palmén Memorial Volume, C. W. Newton and E. O. Holopainen, Eds.. American Meteorological Society, 81-105.

, and P. J. Kocin, 1987: The interaction of jet streak circulations during heavy snow events along the East Coast of the United States. Wea. Forecasting, 1, 289-308.

$\longrightarrow$, R. A. Petersen, C. H. Wash, and K. F. Brill, 1984: The Presidents' Day cyclone of 18-19 February 1979: Synoptic overview and analysis of the subtropical jet streak influencing the precyclogenetic period. Mon. Wea. Rev., 112, 31-55.

—. D. Keyser, K. F. Brill, and C. H. Wash, 1985: President's Day cyclone of 18-19 February 1979: Influence of upstream trough amplification and associated tropopause folding on rapid cyclogenesis. Mon. Wea. Rev., 113, 962-988.

Zwack, P., and B. Okossi, 1986: A new method for solving the quasigeostrophic omega equation by incorporating surface pressure tendency data. Mon. Wea. Rev., 114, 655-666. 